\section{News Updates}

\section{French Team Copies Lab Rats}

By cloning the first rats, a team of French researchers has taken an important step toward producing genetically modified strains of this laboratory workhorse. The lack of embryonic stem cell technology in rats has hampered the production of strains with specific genetic alterations. Cloning could be used as an alternative, but previous attempts to clone rats have been thwarted by the rat oocyte's tendency to begin dividing within an hour of removal from the ovary, leaving insufficient time for researchers to carry out a nuclear transfer procedure. Now, JeanPaul Renard of the National Institute of Agricultural Research (Jouy en Josas, France) and his research team have overcome this problem by collecting oocytes in the presence of MG132, a protease inhibitor that reversibly blocks oocyte activation. Renard's team removed the nuclei from these stabilized eggs and replaced them with nuclei from a fibroblast cell line, and the resulting 129 cloned embryos were implanted into two surrogate mothers, yielding three male pups. One pup died shortly after birth, but the surviving pair grew to sexual maturity and fathered normal pups (Science, published online, doi: 10.1126/science.1088313). Although knockout rats were recently created using random mutagenesis, the ability to clone rats should now allow researchers to generate strains that contain specific genetic alterations.

\section{And Justice for All...Monkeys That Is}

Humans may not be alone in their demand for fair pay, according to a study of brown capuchin monkeys (Cebus apella). Sarah F. Bronson and Frans B.M. de Waal at the Yerkes National Primate Research Center (Atlanta, GA) taught pair-housed female monkeys to trade a token for a food reward. When both monkeys were asked to trade their tokens for pieces of cucumber, the rate of failure was less than 5\%. However, when one monkey was offered a grape-a more highly desired treat-her neighbor often protested by refusing to surrender the token or declining to eat the cucumber (Nature, 18 September). Monkeys were even more likely to protest if they saw another monkey being rewarded without any effort at all. These results suggest that humans' ability to judge fairness is innate rather than learned.

\section{Poodle Genome Revealed}

Man's best friend has joined the human, mouse, and rat on the exclusive list of mammals that have had their genomes sequenced. In the 25 September issue of Science, Ewen Kirkness of the Institute for Genomic Research (Rockville, MD) and colleagues present a draft of the dog genome, which should help researchers unravel the causes of a number of human

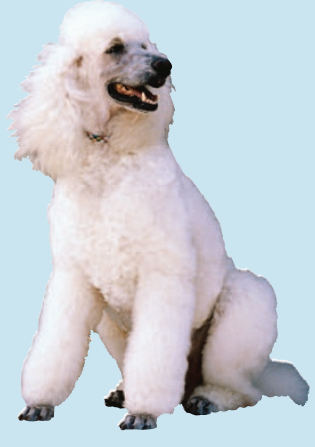

diseases, decipher the evolutionary history of dogs, and allow breeding of healthier dogs. Partial shotgun sequencing of DNA taken from a poodle revealed that the dog genome consists of 2.4 billion base pairs, compared with 2.9 billion base pairs in the human genome, with some of this size disparity resulting from the inclusion in the dog genome of less 'junk DNA' than in its human counterpart. Kirkness' team found 18,473 dog genes with human equivalents. Whereas this draft sequence covers just about $80 \%$ of the dog's genetic makeup, a US government-funded team at the Whitehead Institute (Cambridge, MA) is assembling the full genome of the boxer, which is more genetically inbred than the poodle. 